\title{
Implicación del límite en la enseñanza de la matemática en educación secundaria de Nicaragua
}

\author{
Implication of the limit in the teaching of Mathematics \\ in secondary education in Nicaragua
}

\author{
Winston Joseph Zamora Díaz ${ }^{1}$ \\ Jairo Jóse David Flores Morales ${ }^{2}$ \\ David Gabriel Suárez Rivas ${ }^{3}$ \\ Erling Antonio Trujillo Urbina ${ }^{4}$ \\ Nelson Antonio Pérez Suárez ${ }^{5}$
}

\section{Resumen}

Este artículo es el resultado de una investigación que tuvo por objetivo el análisis de la organización matemática en los libros de textos de octavo y décimo grado de secundaria en Nicaragua sobre el abordaje de aquellos tópicos que estudian funciones racionales y sucesiones. Para el desarrollo de este aporte predominó la investigación documental y el análisis del tratamiento metodológico que los textos hacían a los objetos matemáticos indicados. A partir de los resultados obtenidos, se procedió a elaborar una propuesta que además de evidenciar la organización matemática presentada en los textos, pudiera recoger reflexiones de mejora para la implicación metodológica y didáctica del concepto de límite en la enseñanza de las temáticas. Los principales resultados destacan el hecho de que, en los libros en Nicaragua, la forma en que se propone el abordaje de las funciones racionales y sucesiones en la disciplina de Matemática, presenta limitaciones considerables en materia de organización didáctica, inclusión conceptual de límite y análisis funcional que garantice el correcto desempeño del estudiante en contenidos de educación superior. La propuesta de acción sugerida, es una oportunidad para generar nuevos paradigmas en Educación Matemática en Nicaragua, porque pone a disposición un enfoque didáctico en acuerdo con la gnoseología de la noción del Límite adjunto a los contenidos de funciones racionales y sucesiones.

Palabras clave: Implicación, concepto de límite, organización didáctica, gnoseología.

Doctor en Educación e intervención Social. Profesor de Matemáticas de la Universidad Nacional Autónoma de Nicaragua, UNANManagua E-mail:: winzamora@yahoo.es ORCID: https://orcid.org/oooo-ooo3-2734-3364

2 Doctor en Matemáticas Aplicadas. Profesor de Matemáticas de la Universidad Nacional Autónoma de Nicaragua-UNAN-Managua. Email: jflores@unan.edu.ni

3 Licenciado en Matemática. Profesor de Matemáticas del Ministerio de Educación de Chontales. Email: 10o1davidsn@gmail.com

4 Licenciado en Matemática. Profesor de Matemáticas del Ministerio de Educación de Boaco. Email: trujilloerlingau@gmail.com

5 Licenciado en Matemática. Profesor de Matemáticas del Ministerio de Educación de Boaco. Email: nasp200992@gmail.com

Recibido: 01/11/2017 Aprobado: 01/01/2018

Zamora-Díaz, W., Flores-Morales, J., Suárez-Rivas, D., Trujillo-Urbina, E., \& Pérez-Suárez, N. (2018). Implicación del límite en la enseñanza de la matemática en educación secundaria de Nicaragua. Revista Electrónica de Conocimientos, Saberes y Prácticas, 1(1), 42-53. DOI: https://doi.org/10.30698/recsp.v1i1.3 


\section{Abstract}

This article is the result of an investigation that aimed at the analysis of the mathematical organization in textbooks of eighth and tenth grade of secondary school in Nicaragua on the approach of those topics that study rational functions and successions. For the development of this contribution, documentary research and the analysis of the methodological treatment that the texts made to the indicated mathematical objects predominated. Based on the results obtained, we proceeded to develop a proposal that, in addition to evidencing the mathematical organization presented in the texts, could reflect reflections on improvement for the methodological and didactic implication of the concept of limit in the teaching of the topics. The main results highlight the fact that in books in Nicaragua, the way in which the approach of rational functions and successions in the discipline of Mathematics is proposed, presents considerable limitations in terms of didactic organization, conceptual limit inclusion and functional analysis that guarantees the correct performance of the student in higher education contents. The suggested action proposal is an opportunity to generate new paradigms in Mathematics Education in Nicaragua, because it offers a didactic approach in accordance with the gnoseology of the notion of the limit attached to the contents of rational functions and successions.

Key Words: Implication, concept of limit, didactic organization, gnoseology.

\section{Introducción}

En Nicaragua, se han realizado profundas transformaciones curriculares, metodológicas, tecnológicas en el abordaje de contenidos y en diversas guías de estudio para el docente y estudiantes. Precisamente es en este último aspecto transformado y actualizado que se centra esta propuesta educativa.

Una de las tantas definiciones importantes en el estudio de funciones reales es la de "límite", definición que juega un papel preponderante en el análisis funcional, continuidad de funciones, y derivabilidad. Aspectos que lamentablemente se ven opacados o solapados en los libros de textos que actualmente posee el Ministerio de Educación (MINED) en nuestro país. Esta realidad que enfrenta y en la mayoría de los casos reproduce el docente al abordar temáticas como funciones indeterminadas, funciones racionales y sucesiones, dejan entre ver la necesidad de incluir la noción de límite tanto en los textos de octavo como en undécimo grado.

Es de notar, que en los textos la definición de límite se aborda en forma superficial, evitando en muchas ocasiones dicha noción al tratar de explicar las funciones racionales y el incremento o decremento de las sucesiones, dejando de esta forma vacíos en los aprendizajes estudiantiles. Esta realidad ha servido de motivación para la realización de este trabajo, ya que se considera oportuno dejar de obviar el abordaje 
de la noción de límite en secundaria, previendo así, que en educación superior los estudiantes no posean problemas de aprendizajes en temáticas propias del cálculo diferencial e integral.

De ahí que este trabajo procura, destacar el contexto del proceso de enseñanza aprendizaje en el desarrollo de los contenidos de formas indeterminadas, funciones racionales y sucesiones; describiendo la importancia de la implicación del estudio del límite en dichas temáticas y ofrecer una propuesta educativa para el abordaje de la definición de límite en educación secundaria.

\section{Fundamentación Teórica}

Son muchos los autores que incitan al docente en el nivel que esté, a modificar la forma en que se abordan las funciones racionales. Por ejemplo, Sullivan (1997) en su libro "Precálculo", explica cómo se debe incluir la noción de límite en la enseñanza de funciones racionales al realizar el análisis de la gráfica de una función racional del modo siguiente:

\section{Hacer la gráfica de $H(x)=1 / x^{2}$.}

El dominio de $\mathrm{H}(\mathrm{x})=1 / x^{2}$ consiste en todos los números reales $\mathrm{x}$ excepto o. Por tanto, la gráfica no tiene intersección $-y$, ya que $x$ nunca puede ser igual a cero. La gráfica no tiene intersección $-\chi$, ya que la ecuación $H(x)=0$ no tiene. Por lo tanto, la gráfica de $\mathrm{H}$, no cruzara los ejes de las coordenadas, puesto que:

$$
H(-x)=1 /(-x)^{2}=1 / x^{2}=H(x)
$$

$\mathrm{H}$ es una funcion par, de modo que su gráfica es simétrica respecto al eje y. La tabla 3 muestra el comportamiento de $\mathrm{H}(\mathrm{x})=1 / x^{2}$ para numeros positivos $\mathrm{x}$ seleccionados (usaremos la simetría para obtener la gráfica de $\mathrm{H}$ cuando $x<0$ ). En la tabla 3 , observamos que, conforme los valores de $x$ se aproximan (se acercan) a cero, los valores de $\mathrm{H}(x)$ se incrementan cada vez mas en sentido positivo. Cuando esto pasa decimos que $\mathrm{H}$ no esta acotada en la dirección positiva, lo cual simbolizamos escribiendo $\mathrm{H} \rightarrow \infty$ (se lee $\mathrm{H}$ tiende al infinito). En cálculo el termino límite es utilizado para transmitir estos conceptos, allí usamos los símbolos $\lim _{(x \rightarrow 0)} H(x)=\infty$ que se lee el límite de $\mathrm{H}(x)$ cuando $x$ tiende a cero es igual al infinito, para establecer que $\mathrm{H}(x) \rightarrow \infty$ cuando $(x) \rightarrow \infty$. Observe otra vez la figura 3. Cuando $(x) \rightarrow \infty$ los valores de $H(x)$ se acercan a cero. En cálculo esto es simbolizado $\lim _{(x \rightarrow \infty)} H=0$. La figura 1, ilustra la gráfica" (pp.208-209). 

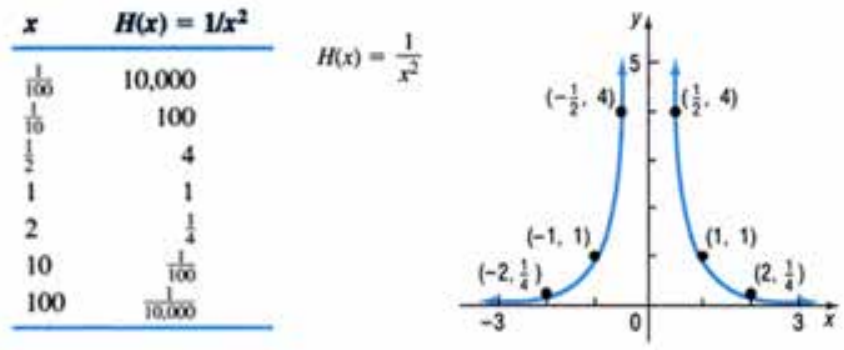

Figura 1: Análisis gráfico de Sullivan al introducir el límite (Sullivan, 1997).

El mismo autor más adelante también utiliza el concepto de límite al referirse a las Asíntotas de una función racional al mencionar que:

"En la figura 2, note que conforme " $\mathrm{x}$ " se vuelve más negativa, esto es, cuando se hace no acotada en la dirección negativa ( $x \rightarrow-\infty$ se lee $x$ tiende a menos infinito) los valores de $R(x)=1 /(x-2)^{2}+1$, tienden a 1 en realidad podemos concluir lo siguiente de la figura 2:

1. Cuando $x \rightarrow-\infty$, los valores de $R(x)$ tienden a 1. $\lim _{(x \rightarrow-\infty)} R(x)=1$

2. Cuando $x \rightarrow 2$, los valores de $R(x)$ tienden al infinito. $\lim _{(x \rightarrow 2)} R(x)=\infty$

3. Cuando $x \rightarrow \infty$, los valores de $\mathrm{R}(\mathrm{x})$ tienden a 1. $\lim _{(x \rightarrow \infty)} R(x)=1$ este comportamiento de la gráfica esta descrito por la recta vertical $x=2$ y la recta $y=1$. Estas rectas son llamadas asíntotas de la gráfica" (Sullivan, 1997, p. 209).

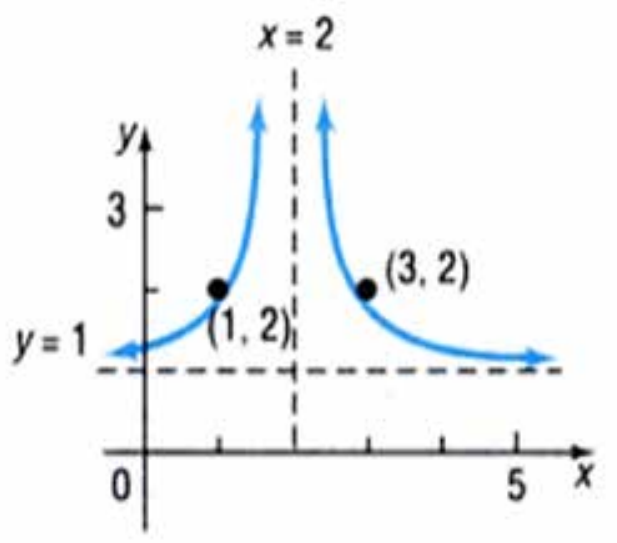

Figura 2: Figura 30C del libro de precálculo (Sullivan, 1997). 
Esta revisión hecha a los planteamientos de Sullivan, pone en evidencia que la información provista por el libro de texto de matemática de 1omo grado de educación secundaria en Nicaragua, es incompleta y carece de un abordaje claro y didáctico. En las definiciones y ejemplos presentados no aparece por ningún lado el límite de funciones como un argumento matemático necesario para entender mejor el comportamiento de las gráficas de las funciones racionales y los puntos que definen indeterminaciones de las mismas.

En la siguiente imagen de la página 279 del libro de texto de décimo grado, se nota como se explica la definición de asíntotas de funciones racionales.

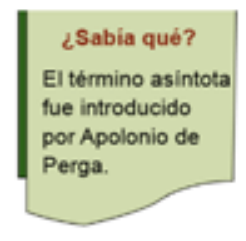

Una asintota de una curva es una recta tal que la distancia entre ésta y la curva tiende a cero cuando ellas tienden a infinito.

La recta $y=b$ es una asintota horizontal de la gráfica de una función $f$ si $f(x)$ se aproxima cada vez más a $b$, cuando $x$ tiende $\mathbf{a}+\infty \quad 0-\infty$.

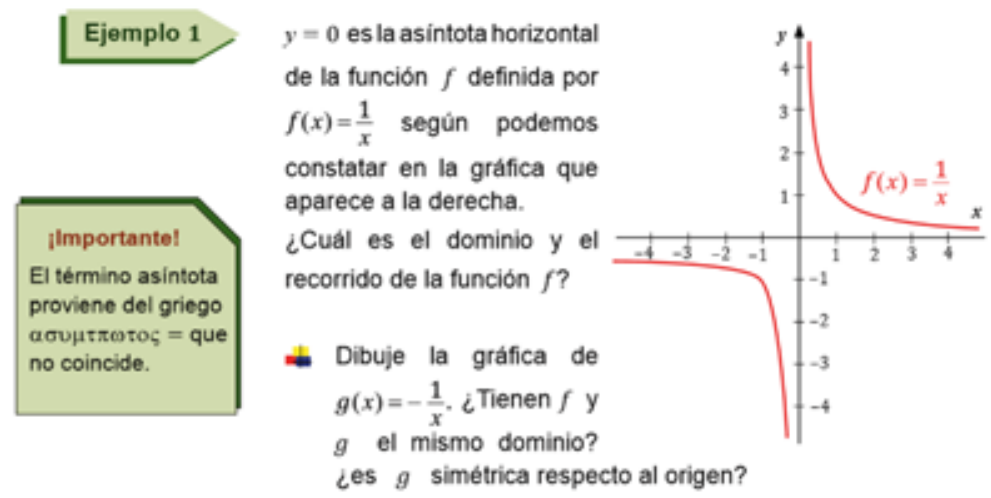

Figura 3: Explicación de asíntota en el libro de texto del MINED.

Lo preocupante en este caso es que el docente que imparte Matemáticas en décimo grado, en la mayoría de las veces lo enseña de manera semejante a como lo sugiere el libro de texto, pues así lo indica la normativa del uso de libros de texto del MINED que expresa: "El uso de los libros de texto... constituirán una de las principales herramientas para construir aprendizajes en niños, niñas, adolescentes y jóvenes" (MINED, 2012, p. 4).

En este mismo libro de texto, a pesar de que se menciona amplia y repetidas veces la tendencia de una variable a tomar un valor especifico real o su tendencia a incrementar o disminuir indefinidamente (al infinito) desde la página 278 a la 291, los autores evitan mencionar o inducir a la noción de limite en algunas de las definiciones propuestas en el texto, así ocurre con la definición de función racional: 


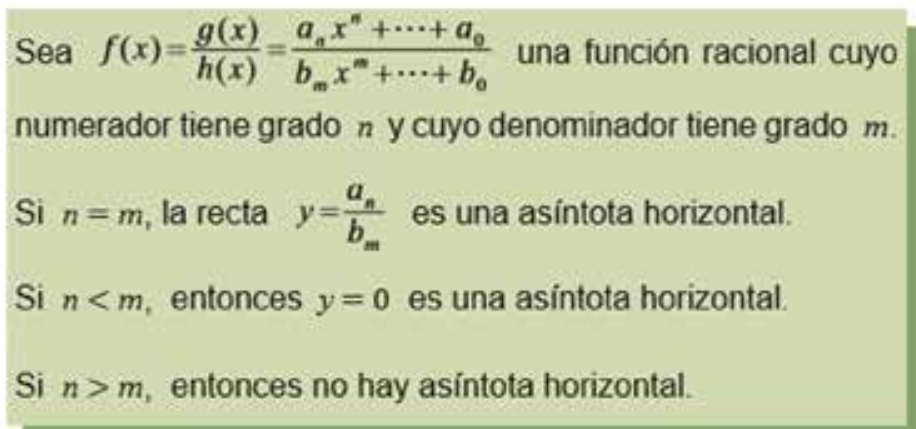

Figura 4: Explicación de tipos de asíntotas en el libro de texto del MINED.

Autores como Priemer y Lazarte (2006), Thomas y Finney (1998), Torroba, Reid y Etcheverry (2006), Ferrante (2009), Plaza (2010) y López (2016); muestran claramente la necesidad de incluir el límite como engranaje crucial para comprender los diversos análisis funcionales que requiere aprender el estudiante en sus estudios. Aspectos que no se logran notar en los libros de textos que actualmente posee el MINED. En forma general se puede citar a Ferrante (2009) que nos indica:

"El concepto de límite funcional forma parte del currículo de educación en la totalidad de las escuelas... Es puerta de entrada al análisis diferencial e integral, y, desde siempre, su enseñanza no ha dejado de preocupar a profesores e investigadores... Hay que partir del hecho de que la comprensión de conceptos como el de límite funcional supone la utilización de estrategias mentales de alto nivel como las que se consideran en el pensamiento matemático avanzado y que la clave reside en la creación de un diseño de enseñanza adecuado a la capacidad y nivel del alumno, que genere un mínimo de interés por el estudio y que le facilite la adquisición de tales conceptos" (p.3).

La recomendación anterior debe calar los planteamientos que generalmente aparecen en los textos nicaragüenses, y que lamentablemente se repiten como una doctrina que no se puede alterar en los centros de educación del país. En forma similar sucede en los contenidos de sucesiones, nuevamente la idea de límite se trata en forma muy superficial. Se puede mencionar por ejemplo que en toda la segunda unidad de sucesiones aritméticas y geométricas del libro de texto de Matemática de undécimo grado, el límite aparece una sola vez al hablar sobre la notación sigma, sin explicar con detalle lo que significa. 


\section{Ejemplo 1}

Solución
Exprese la suma $1^{2}+2^{2}+3^{2}+4^{2}+5^{2}$ utilizando la notación sigma.

Podemos observar que hay cinco sumandos y que lo variable son las bases de cada una de las potencias $1^{2}, 2^{2}, 3^{2}, 4^{2}, 5^{2}$ mientras que los exponentes permanecen iguales a 2 , luego

$$
1^{2}+2^{2}+3^{2}+4^{2}+5^{2}=\sum_{i=1}^{5} i^{2}
$$

El límite inferior es $1 \mathrm{y}$ el superior 5 .

Figura 5: Libro de texto de matemática undécimo grado (MINED, 2012, p. 67).

Para Claros (2010), la relación entre el concepto de límite finito de una sucesión y el concepto de infinito parece a priori debe estar claramente definido así: "en el concepto de límite finito de una sucesión la variable independiente tiende a infinito, y el conjunto formado por los elementos de una sucesión y su límite es el conjunto infinito" (p, 8). Es necesario notar que la inclusión directa del límite para trabajar las sucesiones, hace que el aprendizaje estudiantil de este tópico matemático sea analítico, provechoso y garante de un aprendizaje que le favorecerá en los estudios superiores.

\section{Propuesta o intervención}

Toda propuesta educativa, generalmente nace por la necesidad de cambiar la praxis docente, de incitar al cambio y de facilitar resultados positivos en los estudiantes en materia cognitiva. Como en este trabajo, se ha hecho referencia que actualmente se enseña con libros de textos mal orientados, carentes de didáctica y metodología idónea para garantizar el aprendizaje eficaz de los contenidos (Torrejón, 2016). Por ello, a continuación, se propone una forma alterna de abordar las funciones racionales y las sucesiones en la educación secundaria de nuestro país. 
Tabla 1: Ejemplos de la propuesta educativa para tópicos funcionales y de sucesiones.

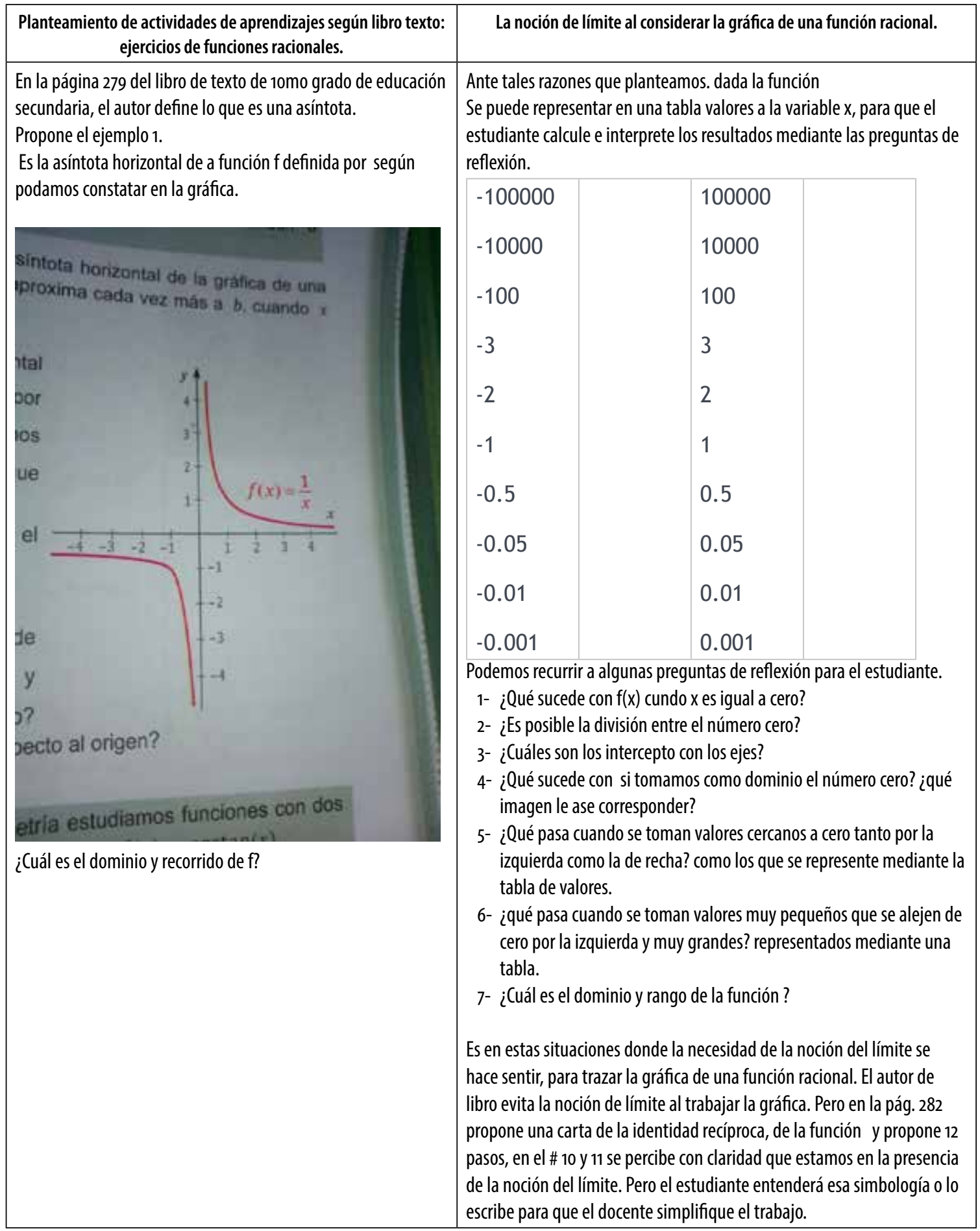




\section{INNOVACIONES EDUCATIVAS}

Tabla 2: Ejemplos de la propuesta educativa para tópicos funcionales y de sucesiones.

Analizaremos ejemplo \# 3 pág. 280.

Identifique las asíntotas horizontales de la función , y describa el comportamiento de es muy grande. Para encontrar la solución de la asíntota horizontal utiliza la división de polinomio. Se hace mención a la noción de límites ya que el autor utiliza la palabra tendencia al infinito.

La grafica es la siguiente y se muestra mediante una fotografía.

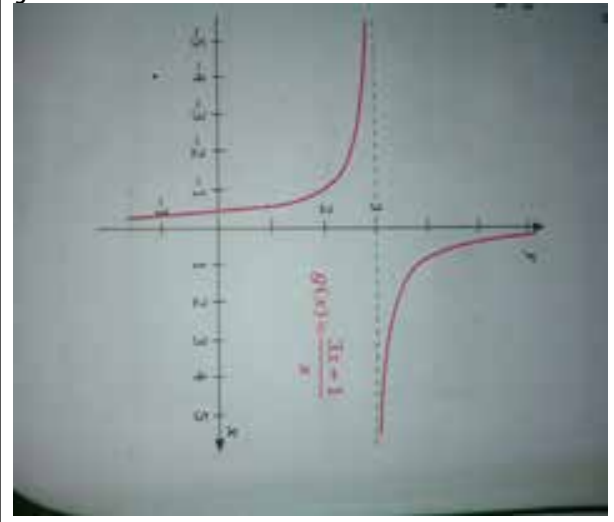

La solución propuesta en el libro se presenta en la siguiente imagen.

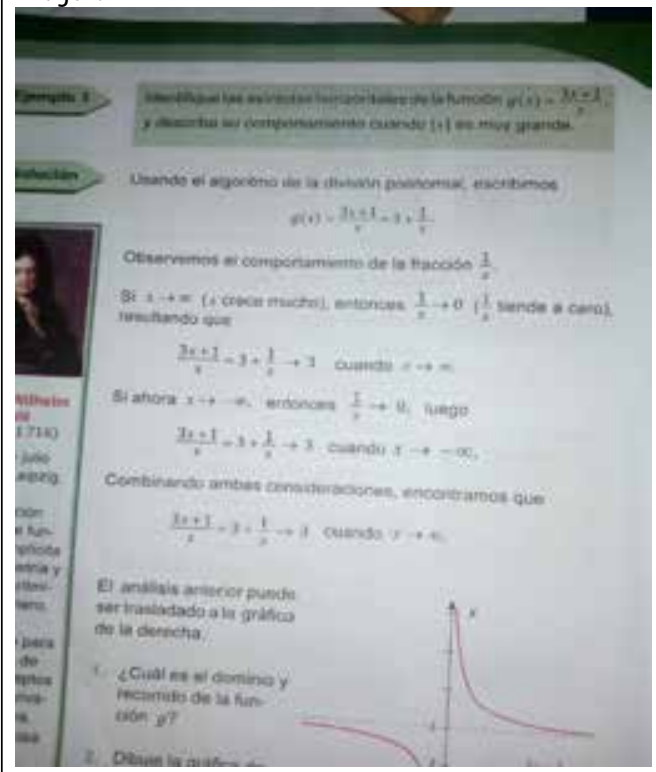

Por tales argumentaciones proponemos. Analizar función racional Podemos realizar varias interrogantes.

1- ¿Qué sucede cuando?

2- ¿como que pasa cuando? ¿qué elemento de imagen me permite obtener este elemento del dominio?

3- ¿Qué sucede con la gráfica cuando tomamos valores muy cercanos a cero negativos como positivos? realizar una tabla de valores.

4- ¿Cuál es el dominio de la función?

5- ¿Cuáles son los intercepto en los ejes?

Pero también argumentamos lo que proponen otros autores en libros muy conocidos.

El límite nos permite tener una visión más clara en el abordaje de las funciones racionales, se puede encontrar con mucha facilidad las asíntotas horizontales y es por lo cual es una necesidad el uso del límite en estos tópicos de educación secundaria.

En el libro de Swokowskiy Cole "Álgebra y Trigonometría con Geometría Analítica" en la pág. 294 nos regala los teoremas de las asíntotas horizontales.

Este libro que es muy bien estructurado utiliza la noción del límite para encontrar asíntotas horizontales, divide cada termino entre la variable de mayor grado, lo cual justifica que si $x$ tiende a ser muy grande es cero.

Además, nos justifica que si el grado del polinomio del numerador como el del denominador son iguales entonces tiene una asíntota horizontal.

¿Cómo encontrar asíntotas horizontales utilizando límite?

Se divide por la literal de mayor grado cada término de la función: $==$ en este caso se considera muy grande como infinito entonces: $===$ es la asíntota horizontal. Esta solución para encontrar asíntotas horizontales, que la división que se presenta en el libro, por tales razones es que lo que se hace una necesidad la implicación de la noción de limite en los estudios de las funciones racionales, para que el estudiante logre mejores conocimientos. 
Tabla 3: Ejemplos de la propuesta educativa para tópicos funcionales y de sucesiones.

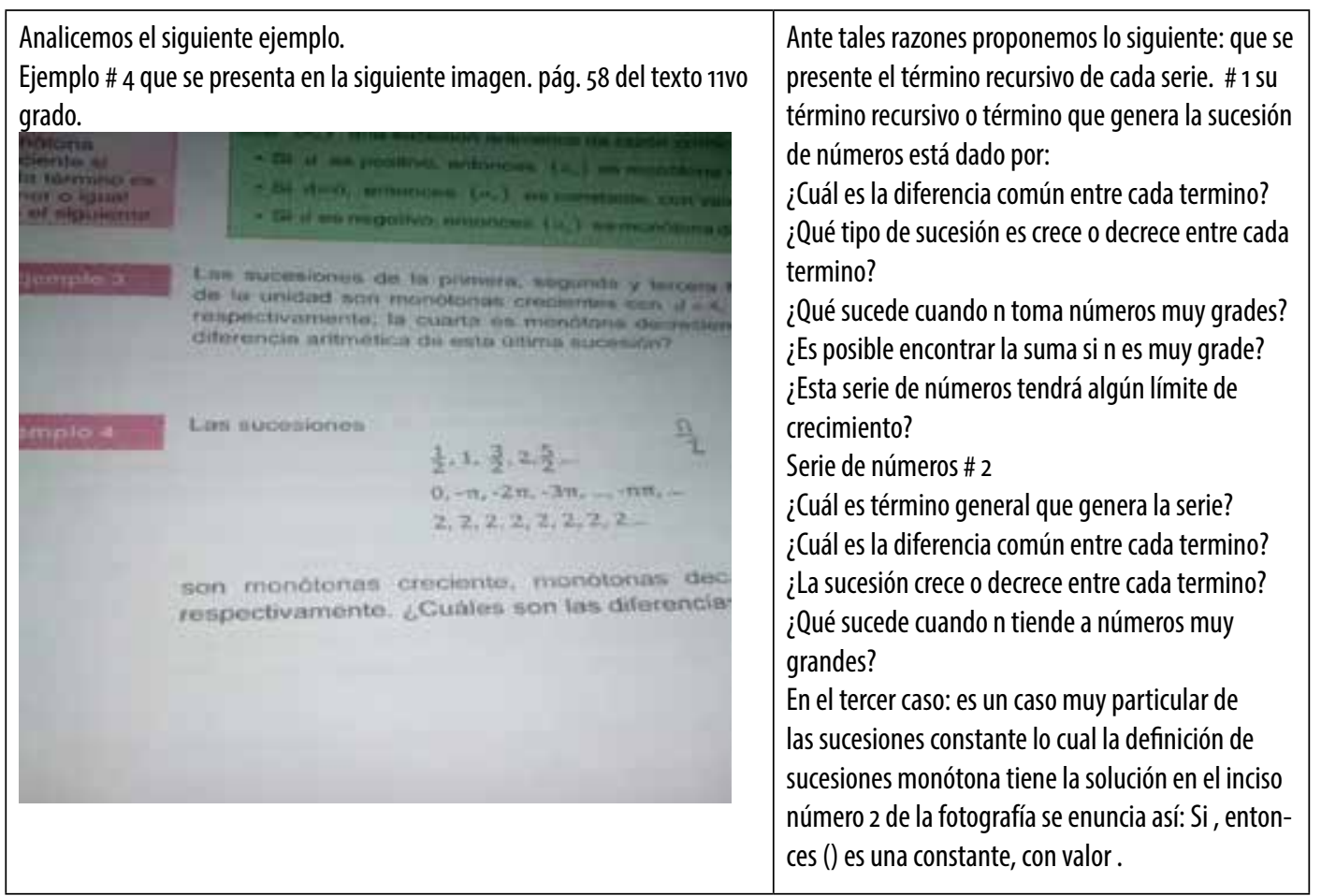

Es importante mencionar que la propuesta didáctica completa estará siendo presentada en la segunda edición del libro "Perspectivas Didácticas para la Enseñanza y Aprendizaje de las Matemáticas", con la finalidad de tener una mejor comprensión de la misma.

\section{Conclusiones y Perspectivas futuras}

En Nicaragua, la forma en que se propone el abordaje de las funciones racionales y sucesiones en la disciplina de Matemática, presenta limitaciones considerables en materia de organización didáctica, inclusión conceptual de límite y análisis funcional que garantice el correcto desempeño del estudiante en contenidos de educación superior.

Se debe tener en cuenta que los autores de los libros de textos, deben en primer lugar poseer además de conocimientos propios de la disciplina, un amplio recorrido en el ámbito metodológico-didáctico, en organizaciones matemáticas que incluyan componentes temáticos que favorezcan aprendizajes relevantes que serán utilizados en niveles de estudios superiores. Las definiciones y conceptos importantes no deben quedar a solapados, confusos y a expensas de la investigación del docente y en el peor de los casos del alumno. Esto debe ser un aporte exigido de un libro de texto que es revisado por profesionales en la materia y aprobado por el MINED. 
El aporte que presentamos, permite llevar a la reflexión la forma en que enseñamos estos tópicos, la repetición de actividades que aparecen en nuestros libros de textos, y la carencia de terminologías importantísimas en esta disciplina que afectan el entendimiento efectivo de los contenidos. Se considera relevante aportar desde nuestra praxis magisterial, investigaciones e innovaciones didácticas una nueva forma de organizar mejor nuestras planificaciones, nuestras explicaciones y evaluaciones.

La propuesta de acción sugerida, es una oportunidad para generar nuevos paradigmas en Educación Matemática en Nicaragua, porque pone a disposición un enfoque didáctico en acuerdo con la gnoseología de la noción del Límite adjunto a los contenidos de funciones racionales y sucesiones.

Por otro lado, se debe fortalecer el trabajo que lleva a cabo el MINED en aras de mejorar la calidad educativa, se necesita realizar una exhaustiva revisión al currículo que contiene los contenidos de matemática en educación secundaria para conseguir una apropiada articulación de los mismos en el proceso de enseñanza-aprendizaje de esta importante disciplina.

\section{Lista de referencia}

Claros, F. (2010). Límite finito de una sucesión: fenómenos que organiza. Granada: UGR.

Ferrante, J. (2009). Una introducción al concepto de límite (dos mil años en un renglón). Buenos Aires: Editorial de la Universidad Tecnológica Nacional.

López, M. (2014). Desarrollo de la comprensión del concepto de límite de una función. Características de trayectorias Hipotéticas de aprendizaje. Alicante: Universidad de Alicante.

MINED. (2012). Matemática undécimo grado. Managua: MINED.

MINED. (2011). Matemática octavo grado. Managua: MINED.

Priemier, N., \& Lazarte, G. (2006). Estrategia para la enseñanza de límite de una función. Jujuy: UNju.

Swokowski. E., \& Cole, J. (2011). Álgebra y trigonometría con geometría analítica. México D.F: CENGAGE Learning.

Sullivan M. (1997). Pre cálculo 4ed. México D.F.: Prentice Hall.

Thomas G. Finney R. (1998). Cálculo de una variable. México D.F: Addison Wesley Longman de México. 
Torrejón, M., \& Caedeñoso Domingo, J. (2016). Propuesta de mejora sobre la didáctica del límite de una función en el aula. Cádiz: UCA.

Torroba, E., \& Reid, M. (2006). Enseñanza-Aprendizaje del concepto de límite de funciones con el uso de TIC. Santa Rosa Argentina: REPEM Memorias. 